\title{
Influence of co-administrated sinomenine on pharmacokinetic fate of paeoniflorin in unrestrained conscious rats
}

\author{
Zhong Qiu Liu ${ }^{\mathrm{a}}$, Hua Zhou ${ }^{\mathrm{a}}$, Liang Liu ${ }^{\mathrm{a}}$, Zhi Hong Jiang ${ }^{\text {a }}$, Yuen Fan Wong ${ }^{\mathrm{a}}$, \\ Ying Xie ${ }^{a}$, Xiong Cai ${ }^{a}$, Hong Xi Xu ${ }^{b}$, Kelvin Chan ${ }^{\mathrm{a}, *}$

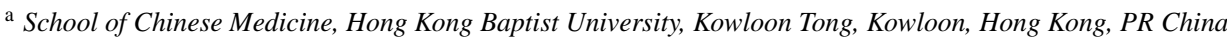 \\ ${ }^{\mathrm{b}}$ Hong Kong Jockey Club Institute of Chinese Medicine Limited, Hong Kong, PR China
}

Received 10 August 2004; accepted 26 January 2005

Available online 20 March 2005

\begin{abstract}
Paeonia lactiflora Pall. (Ranunculaceae) root and Sinomenium acutum Rehder and Wilson (Menispermaceae) stem are two herbs widely used in Chinese medicine to treat rheumatoid arthritis. While, in theory, either herb could be used alone, in practice, Chinese medicine practitioners prescribe them together. Studies on pharmacokinetic interaction between the active constituents of these two herbs (paeoniflorin and sinomenine, respectively) provide empirical evidence to support their clinical practice. A single dose of paeoniflorin ( $150 \mathrm{mg} / \mathrm{kg}) \mathrm{alone}$ and with sinomenine hydrochloride $(90 \mathrm{mg} / \mathrm{kg})$ was administered by gastric gavage to unrestrained conscious male Sprague-Dawley rats $(n=6$, 250-300 g). Blood samples were collected periodically via a jugular vein before and after dosing from $10 \mathrm{~min}$ to $12 \mathrm{~h}$. A high-performance liquid chromatographic (HPLC) assay was developed to determine the plasma concentrations of paeoniflorin. Non-compartmental pharmacokinetic profiles were constructed by using the software PK Solutions 2.0. The pharmacokinetic parameters were compared using unpaired Student $t$-test. After co-administration of sinomenine, the peak plasma concentration of paeoniflorin was elevated $(P<0.01)$, the peak time was delayed $(P<0.01)$, the $\mathrm{AUC}_{0-t}$ was increased $(P<0.001)$, the mean residence time (MRT) was prolonged $(P<0.01)$, the $C_{\mathrm{L}}$ was decreased $(P<0.01)$ and the $V_{\mathrm{d}}$ was reduced $(P<0.05)$. These results indicate that sinomenine hydrochloride at $90 \mathrm{mg} / \mathrm{kg}$ significantly improved the bioavailability of paeoniflorin in rats.

(C) 2005 Elsevier Ireland Ltd. All rights reserved.
\end{abstract}

Keywords: Paeoniflorin; Sinomenine; Rheumatoid arthritis; Pharmacokinetics; Bioavailability

\section{Introduction}

Paeoniflorin is a bioactive monoterpene glucoside (Fig. 1) presenting in the root of Paeonia lactiflora Pall. (family Ranunculaceae), which has been widely used to treat inflammation and arthritic conditions according to the traditional Chinese medical system. The therapeutic effects of the herb and its active component, paeoniflorin, have been confirmed by experimental pharmacological investigations (Takagi and Harada, 1969a,b). However, previous pharmacokinetic studies have shown that paeoniflorin has a poor absorption rate, and thus a very low bioavailability (3-4\%) when adminis-

\footnotetext{
* Corresponding author. Tel.: +852 3411 5308; fax: +852 34115317.

E-mail address: Profchan@hkbu.edu.hk (K. Chan).
}

tered orally. This is probably due to limited transportation of paeoniflorin across the gastrointestinal mucosa (Takeda et al., 1995, 1997). In Mainland China, the total glucosides of Paeonia lactiflora Pall. (TGP) comprising more than $70 \%$ of paeoniflorin, has been approved by the State Food and Drugs Administration of China for the clinical application in treating rheumatic and arthritic diseases as a patented botanical drug (Zhao et al., 1997). While the drug is useful, nevertheless, the low bioavailability of paeoniflorin might be restricting its therapeutic efficacy. Therefore, this investigation aims to use the pharmacokinetic profile of paeoniflorin to find a way to improve its bioavailability and thereby enhance its therapeutic efficacy.

In treating arthritis, Chinese medicine practitioners usually include both the root of Paeonia lactiflora and the 


\begin{tabular}{|c|}
\hline Nomenclature \\
\hline $\begin{array}{l}\mathrm{AUC}_{0-\infty} \text { area under concentration-time curve from } \\
\text { zero up to infinite time }\end{array}$ \\
\hline $\begin{array}{l}\mathrm{AUC}_{0-t} \text { area under concentration-time curve from zero } \\
\text { up to a definite time } t\end{array}$ \\
\hline$C_{\mathrm{L}} \quad$ total clearance \\
\hline$C_{\max }$ maximum plasma concentration \\
\hline$F_{\text {rel }} \quad$ relative bioavailability \\
\hline HPLC high-performance liquid chromatography \\
\hline MRT mean residence time \\
\hline R.S.D. relative standard deviation \\
\hline$t_{1 / 2}$ A phase half-life of absorption phase \\
\hline$t_{1 / 2} \mathrm{D} / \mathrm{A}$ phase half-life of distribution phase \\
\hline$t_{1 / 2} \mathrm{E}$ phase half-life of elimination phase \\
\hline$t_{\max } \quad$ time to reach maximum plasma concentration \\
\hline TGP total glucosides of Paeonia lactiflora Pall. \\
\hline volume of distribution \\
\hline
\end{tabular}

stem of Sinomenium acutum Rehder and Wilson (family Menispermaceae) in their medical prescriptions. This clinical practice suggests that there might be synergic effects between the herbs (Wong and $\mathrm{Wu}, 1936$ ). Previous work on the Chinese medicinal herb Sinomenium acutum demonstrated that the alkaloid sinomenine is one of the main active components of the plant (Tai and Hopkins, 1998). Sinomenine (7,8-didehydro-4-hydroxy-3,7dimethoxy-17-methylmorphinan-6-one; Fig. 2) has been demonstrated to significantly inhibit inflammatory reactions caused by various phlogistic agents (Irino, 1958; Cheng et al., 1964; Huo and Che, 1989). The therapeutic efficacy of sinomenine was also confirmed in patients with rheumatoid arthritis (Ke and Xiu, 1986; Shi et al., 1986). Studies in our previous work have revealed that the alkaloid has marked effects in inhibiting the proliferation of mouse spleen cells, human peripheral blood mononuclear cells as well as rat synovial fibroblasts, the synthesis of proinflammatory mediators of prostaglandin $\mathrm{E}_{2}$ and leukotriene $\mathrm{C}_{4}$ and the production of nitric oxide by macrophages (Liu et al., 1994a,b, 1999). It has also been shown to significantly ameliorate arthritic patholo-

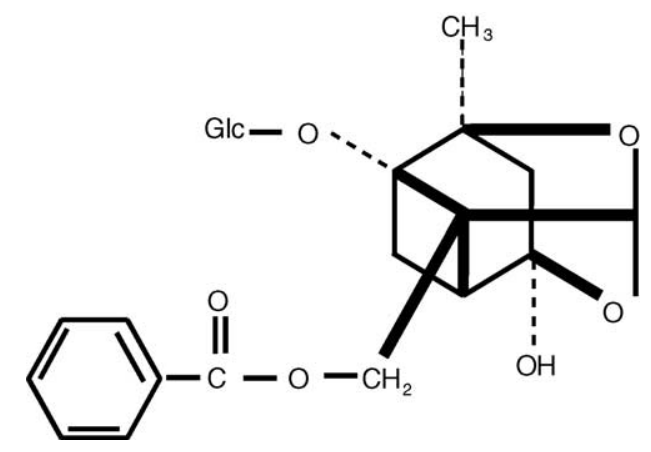

Fig. 1. Chemical structure of paeoniflorin.

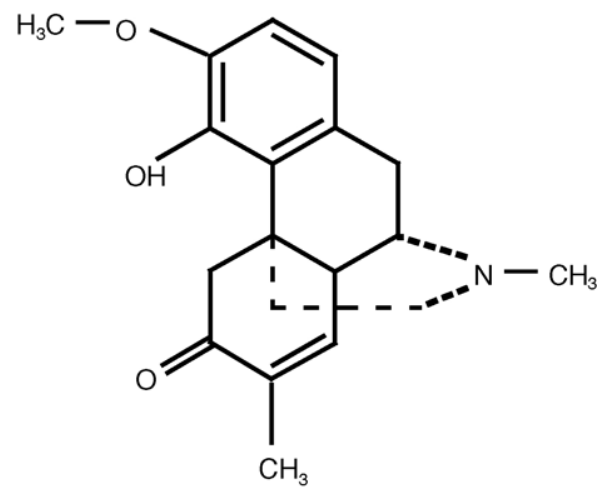

Fig. 2. Chemical structure of sinomenine.

gies in adjuvant arthritis rats (Liu et al., 1999). Studies on the pharmacokinetics of sinomenine using technologies of microdialysis and a hepato-duodenal shunt probe in rats demonstrated that sinomenine undergoes active hepatobiliary elimination; this process might be regulated by $\mathrm{P}$-glycoprotein and probably involves $\mathrm{P} 450$ (Tsai and $\mathrm{Wu}, 2003$ ). Therefore, in the present study we chose to investigate the possible influences of sinomenine on paeoniflorin by pharmacokinetics to see if interaction between these two constituents was responsible for the enhancement observed in using the two herbs together in clinical practice.

\section{Materials and methods}

\subsection{High-performance liquid chromatography analysis}

The HPLC system consisted of an Agilent quaternary HPLC model HP 1100 series (Hewlett-Packard, Palo Alto, CA, USA), fitted with an Altima $\mathrm{C}_{18}$ column $(250 \mathrm{~mm} \times 4.5 \mathrm{~mm}, 5 \mu \mathrm{m})$. The mobile phase used was water:acetonitrile (86:14), filtered through a $0.45 \mu \mathrm{m}$ Millipore filter (Millipore, Hong Kong). The flow-rate was maintained at $1 \mathrm{ml} / \mathrm{min}$, and the detection was performed at a wavelength of $231 \mathrm{~nm}$ under constant temperature $\left(25 \pm 0.1^{\circ} \mathrm{C}\right)$.

\subsection{Chemicals}

The reference standards of paeoniflorin and sinomenine hydrochloride $(\geq 98 \%)$ were purchased from the State Institute for the Control of Pharmaceutical and Biological Products of China. Pentoxifylline, the internal standard for paeoniflorin, was purchased from Sigma (St. Louis, MO, USA). Acetonitrile and perchloric acid were obtained from Merck (Darmstadt, Germany).

\subsection{Animals}

Male Sprague-Dawley rats weighing 250-300 g were purchased from the Laboratory Animal Services Center of the Chinese University of Hong Kong. The animals were housed 
in different cages (five animals per cage) and acclimated in the laboratory for at least one-week prior to testing. Before experiments the animals were fasted for $12 \mathrm{~h}$ with water ad libitum, and maintained at $21{ }^{\circ} \mathrm{C}$ temperature with $60 \%$ relative humidity and a $12 \mathrm{~h}$ light $/ 12 \mathrm{~h}$ dark cycle. After surgery, rats were housed individually in metabolite cages for oneweek recovery and underwent pharmacokinetic treatment according to a jugular-catheterized rat model (Thrivikraman et al., 2002). All procedures involving animals and their care were carried out according to the regulations of the Committee on the Use of Human \& Animal Subjects in Teaching and Research of Hong Kong Baptist University and Health Department of Hong Kong Special Administrative Region.

\subsection{Surgery}

Silicone medical grade tubing $100 \mathrm{~mm}$ long (Silastic Cat. No. 602-155; Dow Corning, Midland, MI, USA) with two stoppers made of medical adhesive silicone (Silastic Cat. No. 801; Dow Corning) was used. For insertion of the catheter in the sinus venosus, the length of the tip of the catheter to the first stopper was set at $26 \mathrm{~mm}$ (Harms and Ojeda, 1974).

Rats were anesthetized by intraperitoneal administration of a mixture of $1.0 \mathrm{ml}$ acepromazine maleate $(10 \mathrm{mg} / \mathrm{ml})$, $4 \mathrm{ml}$ sterile water, $2.5 \mathrm{ml}$ ketamine hydrochloride and $2.5 \mathrm{ml}$ xylazine hydrochloride in a sealed vial at a dosage of $125 \mu \mathrm{l} / 100 \mathrm{~g}$ of body weight. A longitudinal skin incision was made over the area where the right external jugular vein passed dorsal to the pectoralis major muscle. The catheter, filled with 20 units/ml heparinized physiologic saline, was put into the tight jugular vein and then advanced into the sinus venosus. The catheter was inserted up to the first silicone stopper and anchored in place by suturing the stopper to muscle. The free end of the catheter was passed under the skin of the dorsum of the neck just caudal to the ears and attached to the skin, together with a metal spring, which was covered with PVC tubing for protection of the outer part of the catheter. Finally, the catheter was filled with 500 units $/ \mathrm{ml}$ heparinized saline, and a plug was inserted in the free end of the catheter (Cocchetto and Bjornsson, 1983; Yoburn et al., 1984; NRC, 1996; Kohn et al., 1997; Remie, 2000; Thrivikraman et al., 2000).

\subsection{Drug administration and blood sampling}

Catheterized rats were left to recover from surgery in individual metabolite cages for at least 7 days. Before experiments, three types of $1 \mathrm{ml}$ syringes namely, flush syringe, collection syringe and heparinized syringe were prepared. After administration of the test compounds, blood samples were, firstly, drawn from the catheter using a flush syringe fitted with 27-gauge needle until a small amount of blood appeared in the needle bulb, and then the heparin solution was removed from the catheter together with the first sampling of $30-50 \mu \mathrm{l}$ blood. Next, the flushing syringe was removed and replaced by a collection syringe the desired amount $(0.2 \mathrm{ml})$ of blood was taken. Next, the collection syringe was removed and replaced with a heparinized syringe. Blood was gently rinsed from the catheter by flushing with the same volume of heparinized saline ( 20 units $/ \mathrm{ml}$ ) to replace the loss volume of blood a plug was inserted in the catheter. Finally, the blood was expelled from the collection syringe into a heparinized $1.5 \mathrm{ml}$ micro-centrifuge tube.

In each of the experiments, one rat was administered with paeoniflorin alone, while another was administered with paeoniflorin plus sinomenine hydrochloride. Both compounds were prepared as an aqueous solution and administered orally to the fasted rats. For the co-administration protocol, paeoniflorin was first administered at the dosage of $150 \mathrm{mg} / \mathrm{kg}$ body weight, and then, immediately following, sinomenine was administered at the dosage of $90 \mathrm{mg} / \mathrm{kg}$ body weight.

After administration, jugular vein blood samples were collected $(0.2 \mathrm{ml})$ from the rats into heparinized $1.5 \mathrm{ml}$ microcentrifuge tubes at the following time intervals: $0,10,20$, $30,45,60,80,100,120,150,180,240,300,360,540$ and $720 \mathrm{~min}$. Data from these samples were used to construct pharmacokinetic profiles by plotting drug concentrations versus times.

\subsection{Preparation of plasma samples}

Each collected blood sample was immediately transferred into a heparinized $1.5 \mathrm{ml}$ micro-centrifuge tube and centrifuged at $8000 \mathrm{rpm}$ for $6 \mathrm{~min}$ at room temperature. The resulting plasma $(50 \mu \mathrm{l})$ was then mixed with $50 \mu \mathrm{l} 4.5 \%$ perchloric acid aqueous solution and $50 \mu$ internal standard (pentoxifylline in water $4.5 \mu \mathrm{g} / \mathrm{ml}$ ) by vortex for $5 \mathrm{~min}$. The denatured protein precipitate was separated by centrifugation at 13,000 rpm for $10 \mathrm{~min}$ at room temperature. An $80 \mu \mathrm{l}$ volume supernatant was injected into the HPLC system for analysis. For recovery and precision determination of paeoniflorin in plasma, the same procedures for handling the blood samples were used.

\subsection{Calibration curve}

A calibration curve was constructed based on the HPLC analysis of blank rat plasma spiked with various concentrations $(0.22,0.45,0.89,1.78,3.55,7.10$ and $10.65 \mu \mathrm{g} / \mathrm{ml})$ of paeoniflorin, together with a fixed amount of pentoxifylline $(4.5 \mu \mathrm{g} / \mathrm{ml})$. The various concentrations of paeoniflorin in the plasmas were calculated from the values of peak areas by using the equation for linear regression obtained from the calibration curve.

\subsection{Recovery}

Blank rat plasma samples were spiked with four different concentrations $(0.15,0.59,4.73$ and $7.10 \mu \mathrm{g} / \mathrm{ml})$ of paeoniflorin. After preparation of the plasma samples, fixed amounts 
of internal standard (pentoxifylline) were added to the plasma for normalization. The resulting peak areas were compared with those standards carried in distilled water so as to calculate the recovery values.

\subsection{Precision assay}

The precision of the test was determined by triplicate analyses of plasma samples $(n=3)$ spiked with four different concentrations $(0.15,0.59,4.73$ and $7.10 \mu \mathrm{g} / \mathrm{ml})$ of paeoniflorin. To determine intra-daily variances, the assays were carried out on the same samples at three time intervals during one day. Inter-daily variances were also determined by assaying the spiked samples over three consecutive days. Relative standard deviations (R.S.D.) were calculated from these sampled values of paeoniflorin.

\subsection{Pharmacokinetic analysis}

All data were subsequently processed by the pharmacokinetic software, PK Solutions 2.0 (Summit Co., USA). The non-compartmental pharmacokinetic parameters of half-life $\left(t_{1 / 2}\right)$, mean residence time (MRT), area under the plasma concentration-time curve (AUC), clearance $\left(C_{\mathrm{L}}\right)$ and volume of distribution $\left(V_{\mathrm{d}}\right)$ were calculated based on moment methods. Relative bioavailability $\left(F_{\text {ref }}\right)$ of paeoniflorin was calculated using the following formula:

$F(\%)=\frac{\mathrm{AUC}_{0-\infty}(A)}{\mathrm{AUC}_{0-\infty}(B)} \times 100$

where $\mathrm{AUC}_{0-\infty}(A)$ represents the area under the concentration-time curve from zero up to infinite time when paeoniflorin and sinomenine were given jointly and $\mathrm{AUC}_{0-\infty}(B)$ represents the area when paeoniflorin was given alone.

\section{Results}

\subsection{HPLC chromatograms}

The HPLC chromatograms of blank plasma, plasma spiked with paeoniflorin $(4.73 \mu \mathrm{g} / \mathrm{ml})$ and the plasma obtained at $45 \mathrm{~min}$ after oral co-administration of paeoniflorin plus sinomenine together, derived as explained above, are shown in Fig. 3. The retention time of paeoniflorin was found to be around $19.1 \mathrm{~min}$. No interfering peaks were observed within the time frame in which paeoniflorin was detected.

\subsection{Calibration curve}

The calibration curve for area ratio of paeoniflorin to pentoxifylline was linear $\left(r^{2}=0.99997\right)$ over the range of concentrations of $0.15-7.10 \mu \mathrm{g} / \mathrm{ml}$. With the least-squares method, a regression equation of $Y=1.12643 X+0.00135$ ( $X$ is the
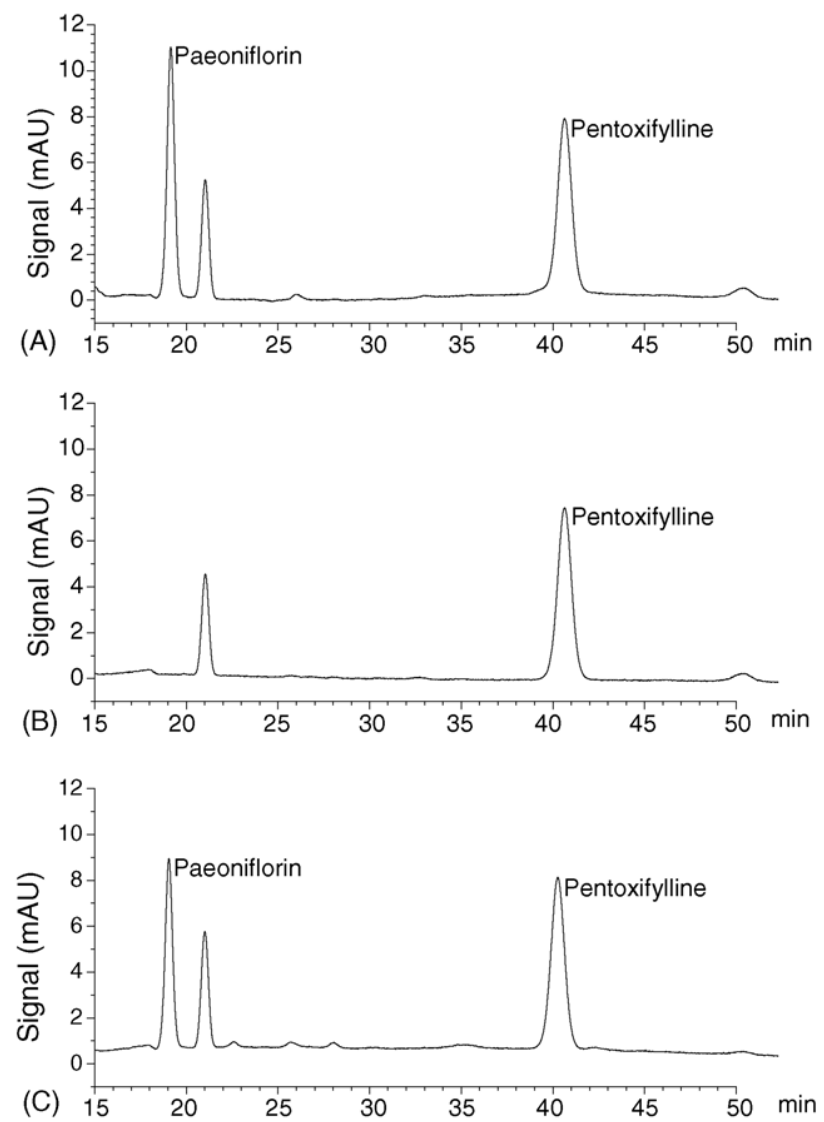

Fig. 3. Chromatograms of paeoniflorin in rat plasma. (A) Plasma sample obtained from rat at $45 \mathrm{~min}$ after oral co-administration of paeoniflorin plus sinomenine together spiked with internal standard (pentoxifylline); (B) blank plasma before oral administration spiked with internal standard (pentoxifylline) and (C) blank plasma spiked with paeoniflorin $(4.73 \mu \mathrm{g} / \mathrm{ml})$ alone and internal standard pentoxifylline $(4.5 \mu \mathrm{g} / \mathrm{ml})$.

amount ratio of paeoniflorin to pentoxifylline in plasma and $Y$ is the area ratio of paeoniflorin to pentoxifylline) was obtained.

\subsection{Recovery test and reproducibility}

The data for validating the HPLC assay are shown in Tables $1-3$. The recovery was higher than $84 \%$ (Table 1 ), the intra-daily variation was lower than $4.22 \%$ (Table 2) and the inter-daily variation lower than $7.69 \%$ (Table 3 ).

\subsection{Determination of paeoniflorin blood plasma of rats}

Fig. 4 shows that, after oral co-administration of paeoniflorin and sinomenine hydrochloride, plasma levels of paeoniflorin were significantly increased with a $C_{\max }$ of $13.7 \mu \mathrm{g} / \mathrm{ml}$.

\subsection{Kinetic analysis}

As calculated from plasma concentrations of paeoniflorin following oral administration of paeoniflorin alone 
Table 1

Recovery of the paeoniflorin assay

\begin{tabular}{llllr}
\hline Test no. & Spiked concentration $(\mu \mathrm{g} / \mathrm{ml})$ & Measured value $(\mu \mathrm{g} / \mathrm{ml})^{\mathrm{a}}$ & ${\text { Recovery }(\%)^{\mathrm{b}}}$ & R.S.D. $(\%)$ \\
\hline 1 & 7.10 & $6.81 \pm 0.14$ & 95.92 & 2.11 \\
2 & 4.73 & $4.62 \pm 0.09$ & 97.67 & 2.01 \\
3 & 0.59 & $0.50 \pm 0.03$ & 84.75 & 6.00 \\
4 & 0.15 & $0.14 \pm 0.01$ & 97.77 & 10.41 \\
\hline
\end{tabular}

${ }^{a}$ Data are expressed as mean \pm S.D. $(n=3)$.

${ }^{b}$ Recovery denotes the ratio of measured value over spiked concentration.

Table 2

Precision of the intra-daily assay

\begin{tabular}{|c|c|c|c|c|c|c|c|}
\hline \multirow[t]{2}{*}{ Test no. } & \multirow[t]{2}{*}{ Spiked concentration $(\mu \mathrm{g} / \mathrm{ml})$} & \multicolumn{3}{|c|}{ Calculated concentration $(\mu \mathrm{g} / \mathrm{ml})$} & \multirow[t]{2}{*}{ Measured value $(\mu \mathrm{g} / \mathrm{ml})^{\mathrm{a}}$} & \multirow[t]{2}{*}{ Precision $(\%)^{\mathrm{b}}$} & \multirow[t]{2}{*}{ R.S.D. (\%) } \\
\hline & & $0 \mathrm{~h}$ & $5 \mathrm{~h}$ & $15 \mathrm{~h}$ & & & \\
\hline 1 & 7.10 & 6.78 & 7.01 & 6.93 & $6.91 \pm 0.12$ & 97.32 & 1.69 \\
\hline 2 & 4.73 & 4.65 & 4.56 & 4.68 & $4.63 \pm 0.06$ & 97.89 & 1.35 \\
\hline 3 & 0.59 & 0.56 & 0.52 & 0.55 & $0.54 \pm 0.02$ & 91.53 & 3.83 \\
\hline 4 & 0.15 & 0.14 & 0.14 & 0.13 & $0.14 \pm 0.01$ & 93.33 & 4.22 \\
\hline
\end{tabular}

${ }^{\text {a }}$ Data are expressed as mean \pm S.D. $(n=3)$.

$\mathrm{b}$ Precision denotes the ratio of measured value over spiked concentration.

Table 3

Precision of the inter-daily assay

\begin{tabular}{|c|c|c|c|c|c|c|c|}
\hline \multirow[t]{2}{*}{ Test no. } & \multirow[t]{2}{*}{ Spiked concentration $(\mu \mathrm{g} / \mathrm{ml})$} & \multicolumn{3}{|c|}{ Treated concentration $(\mu \mathrm{g} / \mathrm{ml})$} & \multirow[t]{2}{*}{ Measured value $(\mu \mathrm{g} / \mathrm{ml})^{\mathrm{a}}$} & \multirow[t]{2}{*}{ Precision $(\%)^{\mathrm{b}}$} & \multirow[t]{2}{*}{ R.S.D. (\%) } \\
\hline & & Day 1 & Day 2 & Day 3 & & & \\
\hline 1 & 7.10 & 6.78 & 6.89 & 7.05 & $6.91 \pm 0.14$ & 97.28 & 1.97 \\
\hline 2 & 4.73 & 4.65 & 4.66 & 4.72 & $4.68 \pm 0.04$ & 98.87 & 0.81 \\
\hline 3 & 0.59 & 0.56 & 0.59 & 0.55 & $0.57 \pm 0.02$ & 96.05 & 3.67 \\
\hline 4 & 0.15 & 0.14 & 0.13 & 0.12 & $0.13 \pm 0.01$ & 86.67 & 7.69 \\
\hline
\end{tabular}

a Data are expressed as mean \pm S.D. $(n=3)$.

$\mathrm{b}$ Precision denotes the ratio of measured value over spiked concentration.

or co-administration with sinomenine hydrochloride to the rats $(n=6$, each group), the pharmacokinetic parameters of paeoniflorin are presented in Table 4. Computation of pharmacokinetic parameters using PK solutions 2.0 software (www.summitpk.com) showed that many parameters (i.e. $t_{\max }, C_{\max }, \mathrm{AUC}, t_{1 / 2}, C_{\mathrm{L}}$ and $V_{\mathrm{d}}$ ) were dramatically altered by co-administration of paeoniflorin plus sinome-

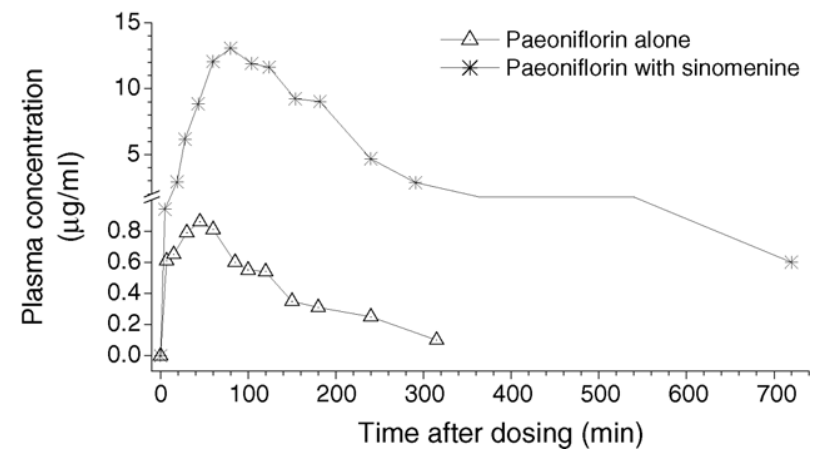

Fig. 4. Plasma concentration-time curve of paeoniflorin. (*) : A plasma concentration-time curve of paeoniflorin in a representative rat after oral coadministration with paeoniflorin $(150 \mathrm{mg} / \mathrm{kg})$ plus sinomenine hydrochloride $(90 \mathrm{mg} / \mathrm{kg}$ ) and $(\triangle)$ : a plasma concentration-time curve of paeoniflorin in a representative rat after oral administration of paeoniflorin alone $(150 \mathrm{mg} / \mathrm{kg})$. nine in comparison with administration of paeoniflorin alone. These results suggest that the pharmacokinetics of the interactions between paeoniflorin and sinomenine might be quite complex.

\section{Discussion}

Pharmacokinetics aims to characterize drug adsorption, distribution, metabolism and excretion, and it is used in the clinical setting to design safe and effective protocols for drug administration. Data and information obtained from pharmacokinetic studies on active compounds of medicinal herbs could help us to understand the complex action of Chinese herbal medicines and to predict a variety of events related to efficacy and toxicity of herbs and herbal preparations (De Smet and Rivier, 1989; De Smet and Brouwers, 1997). Moreover, pharmacokinetic studies of new herbal products could be extremely important as a means to design the most effective therapeutic dosing regimes.

A simple and rapid analytical method is needed for the pharmacokinetic evaluation of paeoniflorin. Our approach to this problem was to use the HPLC method to analyze the concentrations of paeoniflorin in rat's plasma. The validation results showed the R.S.D. of accuracy and precision of the 
Table 4

PK parameters of paeoniflorin after oral administration of paeoniflorin alone $(150 \mathrm{mg} / \mathrm{kg})$ or co-administration of paeoniflorin with sinomenine hydrochloride $(90 \mathrm{mg} / \mathrm{kg})$ to rats $(n=6)$

\begin{tabular}{|c|c|c|}
\hline $\begin{array}{l}\text { Pharmacokinetic } \\
\text { parameters }^{\mathrm{a}}\end{array}$ & Paeoniflorin alone ${ }^{b}$ & $\begin{array}{l}\text { Paeoniflorin plus } \\
\text { sinomenine }^{b}\end{array}$ \\
\hline$t_{\max }(\min )$ & $45.00 \pm 5.00$ & $77.30 \pm 17.50^{\mathrm{c}}$ \\
\hline$C_{\max }(\mu \mathrm{g} / \mathrm{ml})$ & $1.26 \pm 0.23$ & $6.03 \pm 2.45^{\mathrm{c}}$ \\
\hline $\mathrm{AUC}_{0-t}(\mu \mathrm{g} \min / \mathrm{ml})$ & $116.68 \pm 37.54$ & $1528.27 \pm 555.07^{\mathrm{d}}$ \\
\hline $\mathrm{AUC}_{0-\infty}(\mu \mathrm{g} \min / \mathrm{ml})$ & $124.62 \pm 36.91$ & $1540.43 \pm 548.96^{\mathrm{d}}$ \\
\hline$t_{1 / 2} \mathrm{E}$ phase $(\mathrm{min})$ & $55.07 \pm 24.91$ & $84.14 \pm 53.11$ \\
\hline$t_{1 / 2}$ D/A phase $(\min )$ & $19.58 \pm 9.01$ & $53.78 \pm 22.17^{\mathrm{e}}$ \\
\hline$t_{1 / 2}$ A phase $(\min )$ & $17.63 \pm 11.62$ & $44.70 \pm 10.26^{\mathrm{e}}$ \\
\hline$C_{\mathrm{L}}(\mathrm{ml} \mathrm{min} / \mathrm{kg})$ & $1301.83 \pm 429.03$ & $110.44 \pm 45.61^{\mathrm{c}}$ \\
\hline$V_{\mathrm{d}}(\mathrm{ml} / \mathrm{kg})$ & $102044.14 \pm 46608.43$ & $16064.25 \pm 17189.33^{\mathrm{e}}$ \\
\hline MRT (min) & $133.12 \pm 38.63$ & $224.07 \pm 26.62^{\mathrm{c}}$ \\
\hline$F_{\text {rel }}(\%)$ & $\mathrm{N} / \mathrm{a}$ & 12.36 \\
\hline
\end{tabular}

${ }^{\text {a }} C_{\max }$, maximum plasma concentration; $t_{\max }$, time to reach maximum plasma concentration; $\mathrm{AUC}_{0-t}$, area under the concentration-time curve from zero up to a definite time $t ; \mathrm{AUC}_{0-\infty}$, area under the concentration-time curve from zero up to infinite time; $t_{1 / 2} \mathrm{E}$ phase, half-life of elimination phase; $\mathrm{t}_{1 / 2} \mathrm{D} / \mathrm{A}$ phase, half-life of distribution phase; $\mathrm{t}_{1 / 2}$ A phase, half-life of absorption phase; $V_{\mathrm{d}}$, volume of distribution; $C_{\mathrm{L}}$, total clearance; MRT, mean residence time and $F_{\text {rel }}$, relative bioavailability.

b Data are expressed as mean \pm S.D.

c Values are significantly different from that of the paeoniflorin alone group by Student's $t$-test at $P<0.01$.

d Values are significantly different from that of the paeoniflorin alone group by Student's $t$-test at $P<0.001$.

e Values are significantly different from that of the paeoniflorin alone group by Student's $t$-test at $P<0.05$.

paeoniflorin calibration curve were less than $10 \%$, R.S.D. of intra-daily and inter-daily assay were also less than $10 \%$, and the recovery rates were higher than $80 \%$. The results indicated that the HPLC assays used in the present study have good reproducibility, accuracy and precision; they could be successfully applied for the quantitative assay of paeoniflorin in rat blood samples.

In previous reports, the biosamples were obtained by precipitating protein with acetonitrile and extracting with ether to remove non-polar interfering impurities. This is a cumbersome, complex process resulting in rather lower recoveries and involving the use of toxic solvents (Chen et al., 1999; Guan et al., 2003). In our method, $4.5 \%$ perchloric acid aqueous solution was used to denature the plasma protein (Keung et al., 1996). The results of method validation, particularly the recoveries, precision and reproducibility, indicate that the processes for denaturing plasma protein are simple, quick and convenient.

The results of $\mathrm{AUC}_{0-t}$ of pharmacokinetics obtained in the study demonstrate that oral bioavailability of paeoniflorin was elevated by more than 12 times in rats treated with coadministration of sinomenine at the tested doses that are comparable to the doses of these two herbs used in clinical formulas for the treatment of rheumatoid arthritis.

Compared with those of paeoniflorin given alone, the pharmacokinetic parameters of paeoniflorin can be dramatically improved by the concomitant administration with sinomenine; AUC, $C_{\max }, V_{\mathrm{d}}$ and MRT, especially, showed significant differences. The results indicate that sinomenine hydrochloride at a dosage of $90 \mathrm{mg} / \mathrm{kg}$ can markedly elevate the plasma concentration, delay the peak time and consequently increase the bioavailability of paeoniflorin in rats. However, the mechanism of sinomenine on enhancement of the bioavailability of paeoniflorin in rats remains unclear. There are many reports in the literature that support the hypothesis that P-glycoprotein inhibitors enhance drug absorption from the intestine, elevating the bioavailability of the drug (Barthe et al., 1999; Bouer et al., 1999). Previous studies show that sinomenine is a potent inhibitor of immunity (Vieregge et al., 1999), and that its excretion is regulated by $\mathrm{P}$-glycoprotein (Tsai and Wu, 2003); hence, it is possible that sinomenine can alter the disposition, absorption and bioavailability of paeoniflorin. Our preliminary experimental data using a model of rat everted intestinal sacs (Bouer et al., 1999) showed that sinomenine could act as a P-glycoprotein inhibitor, and therefore promote the intestinal transportation of drug from mucosa to serosa, this implied that the enhanced bioavailability of paeoniflorin by the co-administration of sinomenine might be closely related to P-glycoprotein (data not shown).

\section{Conclusion}

In the present study, an HPLC method has been developed and validated for the characterization of plasma, and a jugular-catheterized rat model has been established to investigate the influences of co-administration of sinomenine on the pharmacokinetics of paeoniflorin in freely moving rats. The results show that sinomenine hydrochloride enhanced the plasma concentration and extended the disposition time for paeoniflorin resulting in a marked increase of bioavailability in rats.

\section{Acknowledgement}

This work was supported by a research grant (JCICM6-02) from Hong Kong Jockey Club Institute of Chinese Medicine Limited, Hong Kong.

\section{References}

Barthe, L., Woodley, J., Houin, G., 1999. Gastrointestinal absorption of drugs: methods and studies. Fundamental and Clinical Pharmacology 13, 154-168.

Bouer, R., Barthe, L., Philibert, C., Touraire, C., Woodley, J., Houin, G., 1999. The roles of P-glycoprotein and intracellular metabolism in the intestinal absorption of methandone: in vitro studies using the rat everted intestinal sac. Fundamental and Clinical Pharmacology 13, 494-500.

Chen, L.C., Lee, M.H., Chou, M.H., Lin, M.F., Yang, L.L., 1999. Pharmacokinetic study of paeoniflorin in mice after oral administration of Paeoniae radix extract. Journal of Chromatography B 735, 33-40.

Cheng, S.S., Fu, S.X., Li, Y.S., Wang, N.C., 1964. The pharmacology of sinomenine $\mathrm{I}$ : the analgesic and anti-phlogistic actions and acute toxicity. Acta Pharmacologica Sinica 4, 177-180. 
Cocchetto, D.M., Bjornsson, T.D., 1983. Methods for vascular access and collection of body fluids from the laboratory rat. Journal of Pharmaceutical Sciences 72, 465-492.

De Smet, P.A., Brouwers, J.R., 1997. Pharmacokinetic evaluation of herbal remedies basic introduction, applicability, current status and regulatory needs. Clinical Pharmacokinetics 32, 426-427.

De Smet, P.A., Rivier, L., 1989. A general outlook on ethnopharmacology. Journal of Ethnopharmacology 25, 127-138.

Guan, Y., Li, Y.Z., Li, Y.Y., Guo, H.Z., Guo, D.A., 2003. SPE-HPLC method for the determination and pharmacokinetics studies on paeoniflorin in rat serum after oral administration of traditional Chinese medicinal preparation Guang-Xin-Er-Hao decoction. Journal of Pharmaceutical Analysis 33, 521-527.

Harms, P.G., Ojeda, S.R., 1974. A rapid and simple procedure for chronic cannulation of the rat jugular vein. Journal of Applied Physiology 36, 391-392.

Huo, H.R., Che, X.P., 1989. Study on mechanisms of sinomenine on analgesic and anti-inflammatory activities. Journal of Xian Medical University 4, 346-349.

Irino, S., 1958. Effect of histamine releasers and of anti-inflammatory drugs on the egg-white edema of rat hind paws in relation to skin histamine. Acta Medica Okayama 12, 93-111.

Ke, X.Y., Xiu, M.X., 1986. Treatment of rheumatoid arthritis by sinomenine. Beijing Yi Xuedkjdot 3, 186-187.

Keung, W.M., Lazo, O., Kunze, L., Vallee, B.L., 1996. Potentiation of the bioavailability of daidzin by an extract of Radix puerariae. Proceedings of the National Academy of Sciences of the United States of America 93, 4284-4288.

Kohn, D.F., Wilson, S.K., White, W.J., Benson, G.J. (Eds.), 1997. Anesthesia and Analgesia in Laboratory Animals. Academic Press, San Diego, CA.

Liu, L., Buchner, E., Beitze, D., Schmidt-Weber, C.B., Kaever, V., Emmrich, F., Kinne, R., 1999. Amelioration of rat experimental arthritis by treatment with the alkaloid sinomenine. International Journal of Immunopharmacology 18, 529-543.

Liu, L., Resch, K., Kaever, V., 1994a. Inhibition of lymphocyte proliferation by the anti-arthritic drug sinomenine. International Journal of Immunopharmacology 16, 685-691.

Liu, L., Riese, J., Resch, K., Kaever, V., 1994b. Impairment of macrophage eicosaloid and nitric production by an alkaloid from Sinomenium acutum. Arzneimittel-Forschung 44, 1223-1226.

NRC, 1996. Guide for the Care and Use of Laboratory Animals. National Academy Press, Washington, DC.
Remie, R., 2000. Experimental surgery. In: Krinle, G.J. (Ed.), The Laboratory Rat. Academic Press, San Diego, CA, pp. 523-568.

Shi, P.M., Ma, Z.X., Zhang, W.Z., 1986. Therapeutic efficacy of sinomenine on 60 rheumatoid arthritis case. Xin Yi Xue 3, 292-293.

Tai, Z., Hopkins, S.J., 1998. Sinomenine: antiarthritic anti-inflammatory. Drugs of the Future 23, 45-49.

Takagi, K., Harada, M., 1969a. Pharmacological studies on herb paeony root. I. Central effects of paeoniflorin and combined effects with licorice component Fm 100. Journal of the Pharmaceutical Society of Japan 89, 879-886.

Takagi, K., Harada, M., 1969b. Pharmacological studies on herb paeony root. II. Anti-inflammatory effect, inhibitory effect on gastric juice secretion, preventive effect on stress ulcer, antidiuretic effect of paeoniflorin and combined effects with licorice component Fm 100. Journal of the Pharmaceutical Society of Japan 89, 887-892.

Takeda, S., Isono, T., Wakui, Y., Matsuzaki, Y., Sasaki, H., Amagaya, S., Maruno, M., 1995. Absorption and excretion of paeoniflorin in rats. Journal of Pharmacy and Pharmacology 47, 1036-1040.

Takeda, S., Isono, T., Wakui, Y., Mizuhara, Y., Amagaya, S., Maruno, M., Hattori, M., 1997. In vivo assessment of extrahepatic metabolism of paeoniflorin in rats: relevance to intestinal floral metabolism. The Journal of Pharmacy and Pharmacology 49, 35-39.

Thrivikraman, K.V., Huot, R.L., Plotsky, P.M., 2002. Jugular vein catheterization for repeated blood sampling in the unrestrained conscious rat. Brain Research Brain Research Protocols 10, 84-94.

Thrivikraman, K.V., Nemeroff, C.B., Plotsky, P.M., 2000. Sensitivity to glucocorticoid-mediated fast-feedback regulation of the pypothalamic-pituitary-adrenal axis is dependent upon stressor specific neurocircuitry. Brain Research 870, 87-101.

Tsai, T.H., Wu, J.W., 2003. Regulation of hepatobiliary excretion of sinomenine by P-glycoprotein in Sprague-Dawley rats. Life Sciences 72, 2413-2426.

Vieregge, B., Resch, K., Kaever, V., 1999. Synergistic effects of the alkaloid sinomenine in combination with the immunosuppressive drugs tacrolimus and mycophenolic acid. Planta Medica 65, 80-82.

Wong, K.C., Wu, L.T., 1936. History of Chinese Medicine, second ed. National Quarantine Service, Shanghai, p. 119.

Yoburn, B.C., Morales, R., Inturrisi, C.E., 1984. Chronic vascular catheterization in the rat: comparison of three techniques. Physiology and Behavior 33, 89-94.

Zhao, X.X., Yang, Z.Q., Yang, B.C., Wang, J.R., 1997. Studies on the production technology of anti-rheumatism new drugs-total glucoside of paeony. Natural Product Research and Development 9, 67-70. 